\title{
Results Analysis of Real-Time Edge Detection Techniques using LabView
}

\author{
Htoo Le' Win \\ Department of Electronic \\ Engineering \\ Technological University \\ Thanlyin, Myanmar
}

\author{
Dr. Yin Yin Soe \\ Department of Electronic \\ Engineering \\ Technological University \\ Thanlyin, Myanmar
}

\author{
Daw Yi Yi Lwin \\ Department of Electronic \\ Engineering \\ Technological University \\ Thanlyin, Myanmar
}

\begin{abstract}
Edge detection is the process of finding information of meaningful transition in an image. The purpose of edge detection is to identify basic properties of image like area and shape. The edge detection operators such as canny, sobel, prewitt operators detects the wide range of edges in image. Edge detection is needed to discover the information about the edge and hiding the background data of the video such as the discontinuities in surface orientation and in depth, changes in material properties and variations in scene illuminations. Edge detection is an important basis and significance tasks of image analysis such as boundary detection, image segmentation, motion detection/estimation, texture analysis, object identification, object recognition and stereo matching. Structural Similarity Index (SSIM) is used as a standard among image quality metrics. In this research work, presents the implementation of edge detection of real time video using various edge detection operators (Sobel, Prewitt, Robert, Canny and Mathematical Morphology) with LabView Software, and compares the results with various parameters. The comparisons of experimental results demonstrate the effectiveness of the purposed method.
\end{abstract}

Keywords: SSIM; edge detection; edge detection operators; Mathematical Morphology; LabView

\section{INTRODUCTION}

Edge detection features will include as the important role of object segmentation in machine vision and image processing. Edges are important hints to split region within an object or to identify changes in illumination or color. Edge detection is an initial step in image enhancement procedures to identify the edge of the object, the sudden change in gray level intensity values of neighbor pixel will be an edge, therefore when there is highest difference between two neighbor pixels then there will be an edge is detected. Image edges correspond to points in an image where the gray value changes significantly. In gray scale image each pixel is represented by 8 bit, hence gray level values vary from 0 to 255 , where 0 value stands for black color and 255 value stands for white color [1]. The main purpose of edge detector is to detect both strong as well as weak edges in order to do so various methods. In this research work various edge detector techniques has studied for detecting the edge of objects with SSIM values. SSIM is one of the parameter to evaluate the performance of edge detection method in real time application. Structural Similarity Index is used to assess the similarity between the reference video frame and the test (processed) video frame.

\section{EDGE DETECTION OPERATORS}

\subsection{Mathematical Morphology}

Imperfections may be present in binary images. In particular, the noise and texture will cause distortion to the binary images to the regions produced by simple thresholding. Morphology is nothing but the study of the structure of an object. Morphological filters will simplify the segmented image by smoothing out the outlines of an object using filling small holes and eliminating small projections. Mathematical morphology is a new mathematical technique which is used to process and analyze the images.

One of the applications of Mathematical Morphology is to filter the images; it is a well-known nonlinear filter for image enhancement. It will not use mathematical modeling instead it used set theory to analyze the images. Mathematical Morphology will be well suited for processing binary images because the method will relay on only on the relative ordering of the pixel values not on their numerical values [2]. Morphological operations are generally neighborhood based. The new value for the pixel under inspection (PUI) is determined from the values of its neighboring pixels as shown in Fig. 1. The two main types of binary morphological operations are as follow.

- Erosion: Shrinking the foreground.

- Dilation: Expanding the foreground.

\begin{tabular}{|c|c|c|}
\hline NW & $N$ & $N E$ \\
\hline$W$ & $x$ & $E$ \\
\hline SW & $S$ & $S E$ \\
\hline
\end{tabular}

Figure 1. Pixel Under Inspection (PUI) with Neighboring Pixels

Erosion wears away the edges of image features, decreasing its size, and potentially opening up holes in the object. Dilation refers to the spatial expansion of an object with the potential of increasing its size, filling holes and connecting neighboring objects. Consider the image with the Fig. 1.Simple erosion occurs when the following is applied: if the PUI is 0 , then it retains its value; if it is 1 and all of its cardinal neighbors are also 1 , then set the new pixel value to 1 (otherwise set it to 0) .This is shown in Equation (1).

$x_{\text {new }}=\left\{\begin{array}{lll}0 & x_{\text {old }} & \text { otherwise } \\ 1 & x_{\text {old }}=1 \text { and } \wedge(N, E, S, W)=1\end{array}\right.$

An erosion example is shown in Fig. 2. 


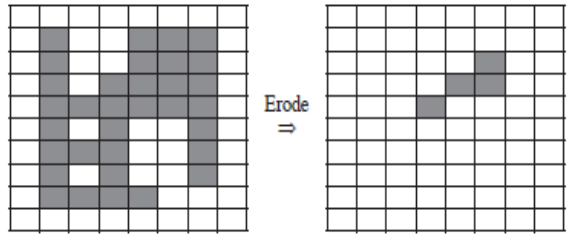

Figure 2. Example of Erosion

Simple dilation occurs when the following procedure is applied: if the PUI is 1 , then it retains its value; if it is 0 , it becomes the logical OR of its cardinal (North, South, East and West) neighbors. This is also present in Equation (2).

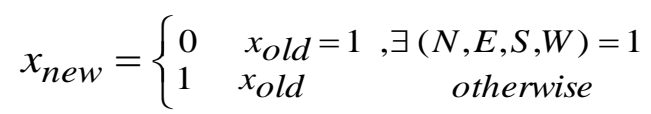

An example is shown in Fig. 3. Note that any PUIs with an original value of 1 are retained, small holes are filled and boundaries are retained [3].
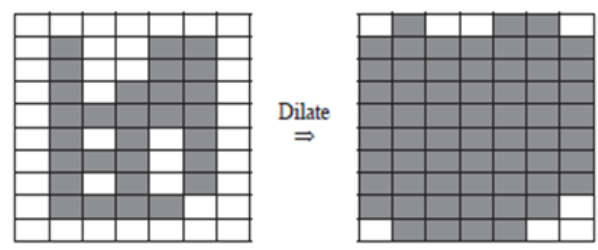

Figure 3. Example of Dilation

\subsection{Traditional Operators}

Some traditional operators are as follow.

- First order derivative/ gradient methods:

Sobel

Prewitt

Robert

- Second order derivative:

Laplacian

Laplacian of Gaussian

Difference of Gaussian

- Optimal edge detection:

Canny edge detection

Edge is a basic feature of an image. Edges define the boundaries between regions in an image and basic step for segmentation and object recognition. An image defined as two dimensional function $\mathrm{f}(\mathrm{x}, \mathrm{y})$, where $x$ and $y$ are spatial coordinates and amplitude of $\mathrm{f}$ at any pair of coordinates ( $\mathrm{x}$, y) defines the light intensity at that point. Edge detection is a process of identifying edges, the sharp change in image pixel intensity. In real world image edges are blurred and have ramp profile, in such cases position of edges are thicker because of blurring effect. Derivative of ramp function is step function, first order derivative is zero at constant gray level and non -zero value along entire ramp region hence magnitude of first order derivative can be used to detect an edge [1].

A gradient based edge detector method includes Roberts, Prewitt and Sobel operator. First order derivative in image processing are implemented using magnitude of gradient. For a function $\mathrm{f}(\mathrm{x}, \mathrm{y})$ the gradient of $\mathrm{f}$ at coordinates $(\mathrm{x}, \mathrm{y})$ is defined as two dimensional column vectors in Equation (3).

$$
\nabla f=\operatorname{grad}(f)=\left[\begin{array}{l}
g_{x} \\
g_{y}
\end{array}\right]=\left[\begin{array}{l}
\frac{\partial f}{\partial x} \\
\frac{\partial f}{\partial y}
\end{array}\right]
$$

This vector points the direction of great rate of change of $f$ at location $(\mathrm{x}, \mathrm{y})$ value at $(\mathrm{x}, \mathrm{y})$ is given by Equation 4 .

$$
\operatorname{mag}(\nabla f)=\sqrt{g_{x}^{2}+g_{y}^{2}}
$$

It is more suitable computationally to approximate the square and square root operation by absolute values [1]. In digital image processing filter marks or operators used to approximate the derivative. Filter masks are convolved with entire image to obtain the edge detected image [4]. $3 \times 3$ filter mask is as shown in Fig. 4.

\begin{tabular}{|l|l|l|}
\hline$Z_{1}$ & $Z_{2}$ & $Z_{3}$ \\
\hline$Z_{4}$ & $Z_{5}$ & $Z_{6}$ \\
\hline$Z_{7}$ & $Z_{8}$ & $Z_{9}$ \\
\hline
\end{tabular}

Figure $4.3 \times 3$ region of an image

The Robert edge detection is introduced by Lawrence Roberts in 1965 . It performs a simple, quick to compute, 2-D spatial gradient measurement on an image. The operator consists of a pair of $2 \times 2$ convolution kernels as shown in Fig. 5 and Fig. 6 . One kernel is simply the other rotated by $90^{\circ}$. This is very similar to the Sobel operator. Horizontal and vertical masks are used to convolve with the entire image to give edge detected image in $\mathrm{x}$ and $\mathrm{y}$ direction respectively.

\begin{tabular}{|l|l|}
\hline $\mathrm{Z}_{1}$ & $\mathrm{Z}_{2}$ \\
\hline $\mathrm{Z}_{3}$ & $\mathrm{Z}_{3}$ \\
\hline
\end{tabular}

Figure 5. $2 \times 2$ region of an image

\begin{tabular}{|c|c|}
\hline+1 & 0 \\
\hline 0 & -1 \\
\hline \multicolumn{2}{|c|}{$G_{X}$}
\end{tabular}

\begin{tabular}{|c|c|}
\hline 0 & +1 \\
\hline-1 & 0 \\
\hline
\end{tabular}

Figure 6. Robert masks

Robert masks of size $2 \times 2$ matric are obtained by following equations:

$$
\begin{aligned}
& G_{x}=\left(Z_{1}-Z_{4}\right) \\
& G_{y}=\left(Z_{2}-Z_{3}\right)
\end{aligned}
$$

\begin{tabular}{|c|c|c|}
\hline-1 & -2 & -1 \\
\hline 0 & 0 & 0 \\
\hline+1 & +2 & +1 \\
\hline
\end{tabular}

\begin{tabular}{|l|l|l|}
\hline-1 & 0 & +1 \\
\hline-2 & 0 & +2 \\
\hline-1 & 0 & +1 \\
\hline
\end{tabular}




\section{$G_{x}$ \\ $G_{y}$ \\ Figure 7. Sobel Operator}

Horizontal derivative approximate as Equation 7 and vertical derivative approximate as Equation 8.

$$
\begin{aligned}
& G_{x}=\left(Z_{7}+2 Z_{8}+Z_{9}\right)-\left(Z_{1}+2 Z_{2}+Z_{3}\right) \\
& G_{y}=\left(Z_{3}+2 Z_{6}+Z_{9}\right)-\left(Z_{1}+2 Z_{4}+Z_{7}\right)
\end{aligned}
$$

The Sobel edge detection method is introduced by Sobel in 1970. The Sobel method of edge detection for image segmentation finds edges using the Sobel approximation to the derivative. It precedes the edges at those points where the gradient is highest. In conjecture at least the operator consists of a pair of $3 \times 3$ complication kernels as given away in under table. One kernel is simply the other rotated by 90 degree. This operator is shown in Fig. 7.

The Prewitt edge detection is proposed by Prewitt in 1970. Prewitt mask is a discrete differentiation operator. This gradient based edge detector is estimated in the $3 \times 3$ neighborhood for eight directions. All the eight convolution masks are calculated. One complication mask is then selected, namely with the purpose of the largest module. Prewitt detection is slightly simpler to implement computationally than the Sobel detection and is used for detecting vertical and horizontal edges in images, but it tends to produce somewhat noisier results [5]. Prewitt operator is shown in Fig. 8.

\begin{tabular}{|c|c|c|}
\hline-1 & -1 & -1 \\
\hline 0 & 0 & 0 \\
\hline+1 & +1 & +1 \\
\hline \multicolumn{3}{|c|}{$G_{x}$}
\end{tabular}

\begin{tabular}{|c|c|c|}
\hline-1 & 0 & +1 \\
\hline-1 & 0 & +1 \\
\hline-1 & 0 & +1 \\
\hline \multicolumn{3}{|c}{$G_{y}$}
\end{tabular}

Figure 8. Prewitt Operator

Horizontal derivative approximate as Equation 9 and vertical derivative approximate as Equation 10.

$$
\begin{aligned}
& G_{x}=\left(Z_{7}+Z_{8}+Z_{9}\right)-\left(Z_{1}+Z_{2}+Z_{3}\right) \\
& G_{y}=\left(Z_{3}+Z_{6}+Z_{9}\right)-\left(Z_{1}+Z_{4}+Z_{7}\right)
\end{aligned}
$$

Canny edge detection method was developed in 1986 by John Canny. The Canny edge detector is an edge detection operator that uses a multi-stage algorithm to detect a wide range of edges in images. The algorithm consists of 4 main stages. The first step involves removing noise with the use of a Gaussian filter. As a result, the obtained image is slightly blurred, without any noises. The next stage features finding the intensity of the image gradient. Considering the fact that edges can be directed in different directions, the algorithm uses four filters to detect the horizontal, vertical, and diagonal edges. Edge operators (e.g. Sobel operator) shall calculate the value of the first derivative of the horizontal and vertical directions. The angle of the edge detection is rounded to four cases representing the horizontal, the vertical and two diagonals (e.g. $0,45,90,135^{\circ}$ ). The obtained contours are characterized by a certain width and it is not the width of one pixel. The third step consists in the elimination of nonmaximal values of pixel intensity. Pixels constituting the orthogonal cross-section of the contour are analyzed, and those lacking maximum intensity values are turned off. The last step includes thresholding aimed at removing unnecessary edges with inclinations below the set threshold. Canny's algorithm can be used in many different environments. Its parameters can be modified so as to enable the algorithm to recognize edges of various features [6].

\section{LABVIEW PROGRAMMING}

LabView is a graphical programming language. Programmers work with two views: front-panel view and a schematic diagram view. Since the front panel view is a necessary part of the integrated Development Environment (IDE), developing human machine Interfaces (HMI) is practically automatic. In fact, it actually is automatic, but the automatically generated HMIs are badly organized. Much of the programming effort goes into "prettying up" the HMI to make it communicate better to humans. Advantages of LabView are as follow.

- Reduced time to discovery - get results faster.

- Reduced time to prototype - create a functional prototype in less time.

- Reduced time to market - productize an idea in less Time.

- Smoother technology transfer process - use the same tools as industry to help achieve a smoother and more efficient technology transfer process.

- Protection of intellectual property - using embedded, field-programmable gate array (FPGA)-based technology.

- Multidisciplinary development - encourage researchers from different disciplines to contribute to the project using the same development tools.

-Improved simulations - achieve better/faster prototyping, hardware-in-the-loop (HIL) simulations, and proofs of concept (POCs) [7][8].

\section{STRUCTURAL SIMILARITY INDEX (SSIM)}

The Structural Similarity (SSIM) index is a method for evaluating the perceived quality of digital images and videos. SSIM is the perceptual metric that quantifies image quality degradation caused by processing. SSIM is easy and well linked with subject evaluation. Due to its popularity, it is often perceptual image and video quality metrics. An early variant was developed in the Laboratory for Image and Video Engineering (LIVE) at the University of Texas at Austin and the full algorithm was developed jointly with the Laboratory for Computational Vision (LCV) at New York University. Structural Similarity is used for measuring the similarity between two images [9]. The Structural Similarity index is a measurement or prediction of image quality based distortionfree image as reference. Peak signal-to-noise ratio (PSNR) and mean squared error (MSE) have proven to be inconsistent with human visual perception. Mean squared error (MSE) and Peak signal-to-noise ratio (PSNR) estimates absolute errors, whereas SSIM is a perception-based model that considers image degradation as perceived change in structural information by incorporating important perceptual phenomena, including both luminance masking and contrast masking. Structural information is the design that the pixels have strong inter-dependencies when they are spatially close. These dependencies carry significant information about the structure of the objects in the visual scene. Luminance masking is a phenomenon whereby image distortions tend to be less visible in bright regions, whereas contrast masking is a 
phenomenon whereby distortions become less visible where there is texture in the image. The SSIM index is calculated on various windows of a frame. The measure between two windows and of common size $\mathrm{N} \times \mathrm{N}$ is

$$
\operatorname{SSIM}(x, y)=\frac{\left(2 \mu_{x} \mu_{y}+C_{1}\right)\left(2 \sigma_{x y}+C_{2}\right)}{\left(\mu_{x}^{2}+\mu_{y}^{2}+C_{1}\right)\left(\sigma_{x}^{2}+\sigma_{y}^{2}+C_{2}\right)}
$$

Where,

- $\mu_{x}, \mu_{y}, \sigma_{x}, \sigma_{y}, \sigma_{x y}$ are the local mean, standard deviations and cross-covariance of image $\mathrm{x}, \mathrm{y}$.

- $\quad C_{1}=\left(k_{1} l\right)^{2}, C_{2}=\left(k_{2} l\right)^{2}$ two variables to stabilize the division with weak denominator

- $\quad l$ is the dynamic range of the pixel-values

- $\quad k_{1}=0.01$ and $k_{2}=0.03$ by default

Similarity condition of SSIM index is defined as;

$$
\operatorname{SSIM}(x, y)=\operatorname{SSIM}(y, x)
$$

In order to assess the image quality, this formula is generally applied on luma, color values or chromatic values. The resultant SSIM index is a decimal value between 0 and 1[10].

\section{PROPOSED SYSTEM}

\subsection{Software requirements}

Software Tool

\section{- LabVIEW 2015}

Software modules

- Vision Acquisition Software

- Vision Development Module

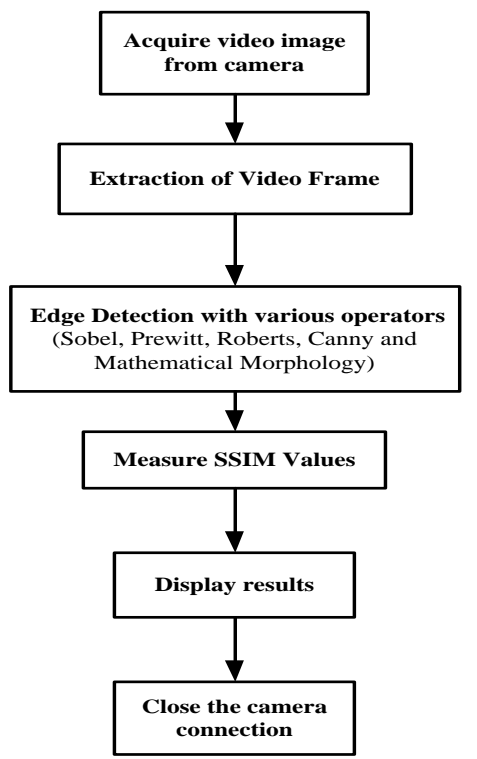

Figure 9. Block Diagram of Proposed system

\subsection{Proposed work}

Opens a camera with Open IMAQdx VI, queries the camera for its capabilities, loads a camera configuration file, and creates a unique reference to the camera. Configures a lowlevel acquisition previously opened with IMAQdx Open
Camera VI. Specify the acquisition type with the Continuous and Number of Buffers parameter and then starts an acquisition. Allocate memory space for acquired images as well as copied images for processing using IMAQ create VI. Images for every loop iteration will be got from specified camera. IMAQ ExtractColorPlanes VI to extract luminance color plane of a color image into an 8-bit image. Edge is detected by using different detection operators. The SSIM values and other parameters are measured. When stop button is hit or an error occurs, running program will be stopped. IMAQ Dispose VI is used to destroy an image, close camera connection and free the space it occupied in memory. Merges error is performed from different functions. After running the algorithm, compare the result between edge detection methods. The block diagram of labVIEW VI for proposed algorithm is shown in Fig. 10

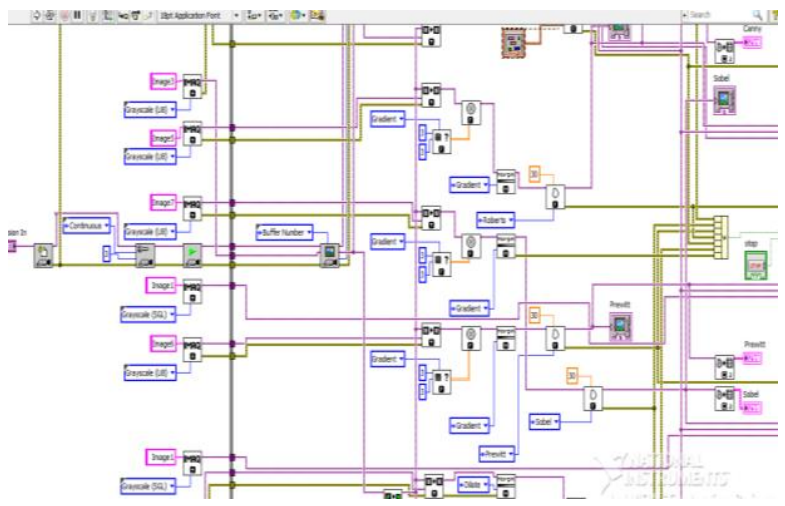

Figure 10. Block Diagram of Proposed Algorithm in LabView

\section{EXPERIMENTAL RESULTS}

This research work describes the relative performance of various edge detection techniques such as traditional edge detection methods (Robert edge detector, Sobel edge detector, Prewitt edge detector), morphological edge detection method (Dilation-Erosion), and optimal edge detection method (Canny edge detector). The edge detection techniques were implemented using LabView 2015, and tested with real time camera image.

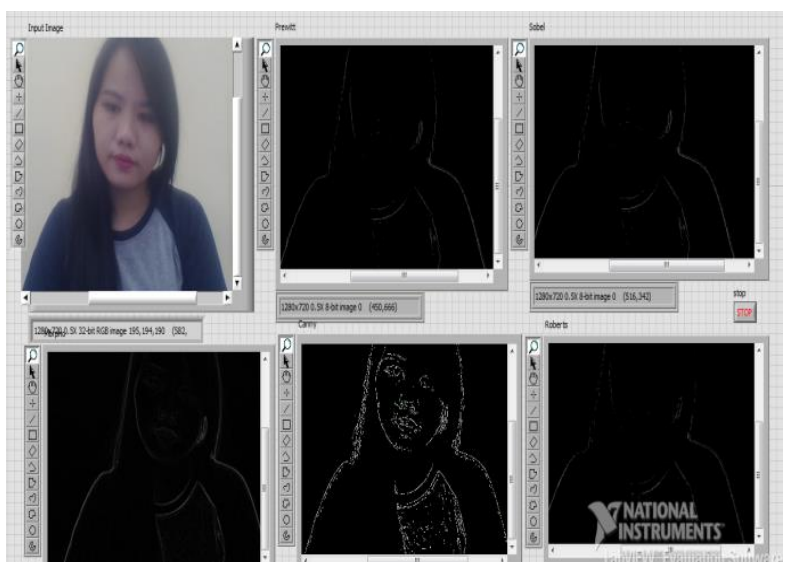

Figure 11. Results of Different Edge Detection (Canny, Morphological, Prewitt, Roberts, Sobel)

In many computer vision and image processing systems the edge detection and corner detection are important tasks. The objective is extracted the principal edge features of the image to produce a clean edge map. It is important to know the differences between edge detection techniques, since edge 
detection is the primary step in object recognition. The intensity of the pixels at the borders of a shadow also translates from a low to a high value. Due to this, any edge detection technique detects this outline of shadows as edges. The original image and the result image obtained by using different edge detection techniques are shown in Fig. 11.

Table 1. Mean SSIM values for Different Operators with other parameters

\begin{tabular}{|c|c|c|c|c|}
\hline & SSIM & $\begin{array}{c}\text { Maximum } \\
\text { Pixel } \\
\text { Intensity } \\
\text { Value }\end{array}$ & Mean & Variance \\
\hline $\begin{array}{c}\text { Mathematical } \\
\text { Morphology }\end{array}$ & 0.047 & 103 & 2.79 & 4.73 \\
\hline Canny & 0.0054 & 255 & 2.97 & 27.36 \\
\hline Sobel & 0.0048 & 142 & 0.41 & 4.92 \\
\hline Prewitt & 0.0047 & 139 & 0.40 & 4.85 \\
\hline Robert & 0.0036 & 155 & 0.26 & 3.79 \\
\hline
\end{tabular}

Mean Structural Similarity Index measurement (SSIM) values are calculated and tabulated in Table. 1 by applying Sobel, Robert, Prewitt, Mathematical Morphology and Canny operators at frame buffer number 100 and described the other parameters. Structural similarity index (SSIM) is able to provide a good approximation of perceived image quality. They are maximum pixel intensity value of edge, the mean and variance of the pixels. An increase in maximum intensity value indicated the enhanced edge. After analysing the results, Suitable edge detection method can be chosen for different purposed works.

\section{CONCLUSION}

A standard webcam and the LabVIEW programme were applied. NI Vision Development tools were used. The programme output was compared during the application of several methods of edge detection. In this research, the most commonly used edge detection techniques of traditional edge detection, morphological edge detection, and optimal edge detection methods. The relative performance of various edge detection techniques is carried out with an image and compared the results under a variety of situations to determine which detector was preferable under different sets of conditions. According to the implement result, the edge detection using mathematical morphology is more efficient than the traditional methods (Canny, Robert, Prewitt and Sobel). Canny's edge detection algorithm performs better than all these operators under almost all scenarios and under noisy conditions, but it is computationally more expensive compared to Sobel, Prewitt and Robert's operator. The Prewitt filter is very sensitive to noise which is a major drawback of it.

\section{ACKNOWLEDGEMENTS}

Firstly, the author would like to acknowledge Dr. Thein Gi, Rector of Technological University (Thanlyin), for her kind permission to carry out this research work. The authors would like to thank many colleagues from digital image processing research group of Department of Electronic Engineering of Technological University (Thanlyin). The author particularly wishes to acknowledge all the teachers from Department of Electronic Engineering, Technological University (Thanlyin), for their support, encouragement and invaluable guidance in preparation of this research. The authors would like to express their thanks to all persons who have given support during the preparation period of this research work.

\section{REFERENCES}

[1] R. C. Gonzalez, R. E. Woods, Digital Image Processing. $3^{\text {rd }}$ ed, Prectice Hall, 2007, pp. 187-190.

[2] Panchami S V, Dr. M V Sathyanarayana, Senthilkumar S, "Continuous Image Acquisition and Edge Detection Using Morphological Filters and Classical Edge Detection Algorithms in Labview", International Journal of Engineering Research \& Technology, vol. 6, no. 12, 2017, pp. 242-245.

[3] Christopher G. Relf, “ Image Acquisition and Processing with LabVIEW", ch. 6.

[4] Girish N. Chaple, R. D. Daruwala, Manoj S. Gofane, "Comparisions of Robert, Prewitt, Sobel operator based edge detection methods for real time uses on FPGA", International Conference on Technologies for Sustainable Development, February 2015, pp. 04-06.

[5] Muthukrishnan.R, M.Radha, "edge detection techniques for Image segmentation", International Journal of Computer Science \& Information Technology, vol. 3, no. 6, 2011, pp. 259267.

[6] Kamil Sidor, Anna Szlachta, "The Impact of the Implementation of Edge Detection Methods on the Accuracy of Automatic Voltage Reading", Measurement Science Review, vol.17, no. 2, 2017, pp. 93-99.

[7] NI Vision for LabVIEW User Manual.

[8] Ravi Kumar A.V, Nataraj K.R, "Result Analysis of LabVIEW and MatLab in Application of Image Edge Detection", International Journal of Computer Applications, vol. 48, no. 9, 2012, pp. 6-10

[9] Journal of Advanced Research in Computer and Communication Engineering, vol. 2, no. 1, January 2013.

[10] S.Dutta and BB.Chudhuri, "A color edge detection algorithm in RGB color space," Internal conference on Advances in Recent Technologies in communication and computing, 2009, pp.337340 . 